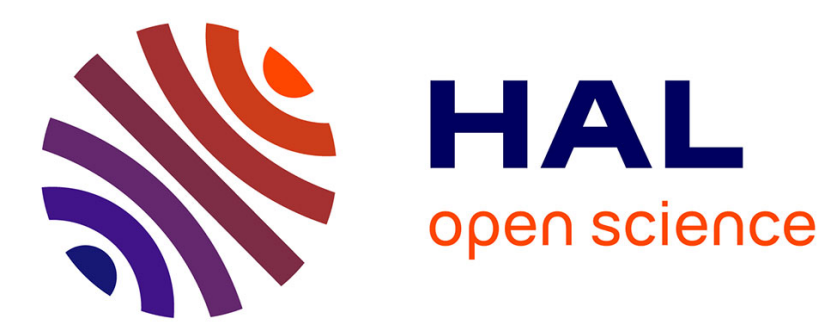

\title{
Comptabilisation des écarts actuariels. Mise en perspective des pratiques françaises
}

\author{
Samira Demaria
}

\section{To cite this version:}

Samira Demaria. Comptabilisation des écarts actuariels. Mise en perspective des pratiques françaises. La Revue des Sciences de Gestion, 2010, Septembre-Décembre (245-246), pp.111-118. halshs00585681

\section{HAL Id: halshs-00585681 https://shs.hal.science/halshs-00585681}

Submitted on 6 Dec 2013

HAL is a multi-disciplinary open access archive for the deposit and dissemination of scientific research documents, whether they are published or not. The documents may come from teaching and research institutions in France or abroad, or from public or private research centers.
L'archive ouverte pluridisciplinaire HAL, est destinée au dépôt et à la diffusion de documents scientifiques de niveau recherche, publiés ou non, émanant des établissements d'enseignement et de recherche français ou étrangers, des laboratoires publics ou privés. 


\title{
Comptabilisation des écarts
}

\section{actuariels}

\section{Mise en perspective des pratiques françaises}

\author{
Samira Demaria \\ Maître de Conférences en Sciences de Gestion \\ Université de Nice-Sophia Antipolis \\ GREDEG CNRS UMR 7321 \\ 250 rue Albert Einstein 06560 Valbonne \\ 0493954385 \\ demaria@gredeg.cnrs.fr
}




\section{Comptabilisation des écarts actuariels : Mise en perspective des pratiques françaises}

Résumé : Cet article poursuit deux objectifs : d'une part, identifier, sur une période de cinq exercices, les pratiques des groupes français cotés en matière de comptabilisation des écarts actuariels et d'autre part, mettre en perspective l'évolution des pratiques observées grâce aux apports de la théorie néo-institutionnelle sociologique.

Mots-clés : Choix d'options comptables, IAS 19, écarts actuariels, théorie néo-institutionnelle sociologique

\section{Actuarial gap recognition: some French evidence}

Abstract: The paper follows two main goals: in the one hand it aims at identifying the method of recognition of actuarial gains and losses retained by French listed groups during a five years period. On the other hand it aims at explaining the evolution of observed practices thanks to the contribution of the neo-institutional theory.

Keywords : Accounting choices, IAS 19, actuarial gains and losses, neo-institutional theory

\section{Contabilización de diferencias actuariales: presentación de las practicas francesas}

Resumen : Este papel tiene dos objetivos: en una primera parte, identifica, en cinco ejercicios, las practicas de grupos francés famosos por su contabilidad de diferencias actuariales y en secunda parte, presenta la evolución de las practicas observadas gracia a las aportaciones de la teoría neo-institucional sociológica.

Palabras clave: Elección d'opciones contables, IAS 19, diferencias actuariales, teoría neoinstitucional sociológica 


\title{
Comptabilisation des écarts actuariels
}

\author{
Mise en perspective des pratiques françaises
}

\section{INTRODUCTION}

L'application des normes comptables internationales IAS/IFRS ${ }^{1}$ a eu comme conséquence l'introduction d'options en matière d'évaluation et de comptabilisation de certaines opérations économiques. Cet article se focalise sur les options permises par la norme IAS 19 «Avantages du personnel» concernant la comptabilisation des pertes et gains actuariels. La volonté de centrer la réflexion sur cette norme repose sur son intérêt éminemment stratégique. D'une part, comme le souligne Sir David Tweedie (IASB, 2008, p.2), «la comptabilisation des retraites est un champ complexe et d'une très grande importance. Le montant des engagements de retraite des quatre-vingt plus grandes firmes mondiales est estimé à 700 milliards de livres. D'ailleurs, pour certains groupes, le montant de la dette des retraites est supérieur à la valeur de la capitalisation boursière ». D'autre part, Alexis Canuet (2007) souligne l'importance de l'impact de la comptabilisation des engagements de retraite dans les groupes français. Il met en évidence le sous-provisionnement chronique, compte tenu de la valeur actuelle, des groupes français en matière de retraite.

La comptabilisation des engagements de retraite supposant l'établissement d'hypothèses quant au montant à enregistrer, dès lors, des écarts sont possibles. C'est justement pour la comptabilisation de ces écarts que l'International Accounting Standard Board (IASB) offre plusieurs options. Actuellement, IAS 19 et son amendement permettent aux groupes d'appliquer soit une comptabilisation intégrale au résultat, soit la méthode du corridor, soit la méthode dite SoRIE (Statement of Recognised Income and Expense) consistant à imputer directement aux capitaux propres les pertes ou gains actuariels de l'exercice.

Cet article vise à identifier les pratiques retenues par les groupes français appartenant au SBF 120 sur la période 2004 à 2008. L'observation des choix effectués nous conduit à nous interroger sur les raisons du recours accru à la méthode SoRIE. Afin de comprendre cette

\footnotetext{
${ }^{1}$ Les normes comptables internationales sont composées des International Accounting Standards (IAS) et des International Financial Reporting Standards (IFRS). Lorsque nous écrivons IAS/IFRS, nous faisons référence globalement à toutes les normes internationales. L'usage du terme IFRS seul, dans les différents écrits professionnels et académiques, renvoie lui aussi aux normes en général. Cependant, lorsque nous indiquons IAS, nous parlons des normes promulguées avant 2001 référence globalement à toutes les normes internationales.
} 
tendance, nous avons retenu la théorie néo-institutionnelle sociologique comme cadre d'interprétation des choix d'options comptables.

La première section est consacrée à la présentation du contexte de la recherche, à la fois réglementaire et théorique. La deuxième section vise quant à elle à expliquer les méthodes de collecte des données ainsi qu'à restituer les observations. Enfin, la troisième section propose une explication de l'objet de recherche au travers du prisme néo-institutionnel sociologique.

\section{LE CONTEXTE DE LA REFLEXION}

Nous présentons dans cette section le cadre de la recherche : d'une part, nous précisons le contexte réglementaire régissant la comptabilisation des engagements de retraite, en particulier le cas des écarts actuariels, et d'autre part, nous décrivons le cadre théorique de la réflexion.

\subsection{LE CONTEXTE REGLEMENTAIRE}

La norme IAS 19 traite des méthodes de comptabilisation et d'évaluation des avantages du personnel. Ceux-ci sont définis par l'IASB comme : «toutes formes de contrepartie donnée par une entité au titre des services rendus par son personnel ${ }^{2} »$ (IAS 19 §7). La norme identifie plusieurs catégories d'avantages du personnel (IAS $19 \S 4)^{3}$, dont les avantages dits postérieurs à l'emploi qui doivent faire l'objet d'une comptabilisation spécifique. Nous ne rentrerons pas dans le détail des différents types de rémunération pour nous centrer sur les pensions postérieures à l'emploi à prestations définies, car c'est pour la comptabilisation de ces dernières que l'IASB autorise trois options. Les régimes à prestations définies apparaissent lorsque l'employeur s'engage à verser des avantages sous forme de pensions ou d'indemnités de départ à la retraite, en fonction de l'ancienneté de l'employé et de son salaire, le risque actuariel et le risque des placements incombant à l'entreprise. Dans ce cas, régi par IAS 19, un engagement de retraite doit être calculé. L'IASB exige que ces engagements soient déterminés selon la méthode dite prospective. Celle-ci établit le coût des prestations attribuables aux bénéficiaires d'un régime au titre des années de service effectuées jusqu'à la

\footnotetext{
2 Les stock-options sont exclues des avantages du personnel et ne sont pas traitées par IAS 19 mais conformément à la norme IFRS 2.

${ }^{3}$ Les avantages à court terme comme les salaires ; les avantages postérieurs à l'emploi comme les prestations de retraites ; les indemnités de fin de contrat de travail et les autres avantages à long terme, notamment les congés liés à l'ancienneté.
} 
date d'évaluation de l'engagement. Le coût des droits acquis doit ensuite être modulé en fonction de l'espérance de vie, du turn over de l'entreprise et de la politique salariale. Cependant, des écarts entre les hypothèses actuarielles retenues et les réalisations sont inévitables. Ces écarts doivent être comptabilisés dès l'exercice qui suit leur constatation. C'est à ce niveau que des options existent. Pour la comptabilisation des écarts actuariels liés aux engagements de retraite, trois méthodes sont proposées par l'IASB :

- L'enregistrement au compte de résultat selon la méthode du corridor (IAS 19 §92) : pour l'évaluation du passif au titre des prestations définies, l'entreprise doit comptabiliser une fraction de ses écarts actuariels en produits ou en charges, si les écarts actuariels cumulés non comptabilisés à la fin de l'exercice précédant excèdent la plus grande des deux valeurs ci-dessous :

○ $10 \%$ de la valeur actuelle de l'obligation au titre des prestations définies à la date de clôture (avant déduction des actifs du régime) ;

○ $10 \%$ de la juste valeur des actifs du régime à la date de clôture.

- L'enregistrement intégral au compte de résultat: une entreprise peut adopter toute méthode conduisant à comptabiliser de façon systématique tous les écarts actuariels plus rapidement, sous réserve d'appliquer la même base de comptabilisation pour les gains et pertes actuariels et de l'appliquer de façon permanente d'un exercice à l'autre (IAS 19 $\S 93)$.

- L'enregistrement intégral en capitaux propres selon la méthode SoRIE (IAS 19 amendée §93a) : une entité peut décider de comptabiliser les écarts actuariels dès leur apparition, en dehors du compte de résultat, autrement dit, directement en capitaux propres. Le montant des écarts actuariels doit être présenté dans un tableau spécifique des produits et charges comptabilisés.

Il faut noter que l'option retenue doit être appliquée à l'ensemble des écarts actuariels et pour tous les régimes à prestations définies du groupe.

La réglementation française reconnaît l'existence des engagements de retraite, ainsi la recommandation du Conseil National de la Comptabilité (CNC) 2003 R-01 fixe une méthodologie d'évaluation et de comptabilisation proche du corridor. Toutefois, comme le souligne la RF Comptable (2003), la constatation des passifs liés aux retraites n'est pas obligatoire. En effet, l'article 123-13 du code de commerce ${ }^{4}$ laisse la faculté aux entreprises

\footnotetext{
${ }^{4}$ «Le montant des engagements de l'entreprise en matière de pension, de compléments de retraite, d'indemnités et d'allocations en raison du départ à la retraite ou avantages similaires des membres ou associés de son
} 
de ne pas comptabiliser un passif pour les engagements de retraite à la condition que le montant global en matière de pension soit indiqué en annexe. Selon l'AMF (2004), la norme IAS 19, et plus spécifiquement la problématique des engagements de retraite, est l'une des options susceptible d'avoir les impacts les plus importants sur les comptes des sociétés françaises.

Enfin, il faut noter que la norme IAS 19 est jugée obsolète et fait actuellement l'objet d'un projet de révision conjoint entre l'IASB et le Financial Accounting Standard Board (FASB organisme de normalisation des États-Unis). Pour Alexandra Pétrovic (2008), la norme ne répond pas à l'objectif de comparabilité des états financiers au regard du nombre trop important d'options qu'elle autorise. Un discussion paper a été publié courant $2008^{5}$ et l'IASB souhaite parvenir à une norme révisée en 2011. Les enjeux de cette norme conjointe sont particulièrement stratégiques au regard de la reconnaissance des normes IAS/IFRS sur les marchés américains, ainsi que de leur éventuelle adoption par les États-Unis à l'horizon 2014. Évoquant la volonté d'accroître la transparence et la comparabilité des états financiers, l'IASB entend à terme supprimer l'option permettant de différer l'enregistrement des écarts actuariels, c'est-à-dire la possibilité de constater les gains et pertes actuariels selon la méthode du corridor. Cette volonté est approuvée par le CNC (2008) et par les instances européennes qui sont favorables à l'élimination du corridor afin de favoriser la reconnaissance immédiate des engagements de retraite (Danielle Sougné, 2009). Les travaux en cours au sein des organismes de normalisation font état de différentes approches afin de réformer la comptabilisation des écarts actuariels. Celle qui semble être privilégiée reposerait sur la suppression des options et l'imposition d'une méthode de comptabilisation mixte entre le bilan et le compte de résultat (Cécile Darche et Emmanuelle Levard-Guilbault, 2009). Il sera donc intéressant de se pencher sur la manière dont les firmes vont prendre en compte ces évolutions normatives en cours.

personnel et de ses mandataires sociaux est indiqué dans l'annexe. Par ailleurs, les entreprises peuvent décider d'inscrire au bilan, sous forme de provision, le montant correspondant à tout ou partie de ces engagements ».

${ }^{5}$ L'IASB a publié le 7 avril 2008 le Discussion Paper Preliminary Views on Amendments to IAS 19 Employee Benefits. Après prise en compte des commentaires reçus courant septembre 2008, l'IASB souhaite publier un exposure draft au troisième trimestre 2009. 


\subsection{LA THEORIE NEO-INSTITUTIONNELLE SOCIOLOGIQUE}

Les recherches sur les choix comptables usent fréquemment de la théorie politicocontractuelle comme cadre d'explication des pratiques observées ${ }^{6}$. Ces travaux tentent d'expliquer les choix selon les caractéristiques politiques (taille, cotation aux États-Unis) et contractuelles (particularités des contrats de rémunération des dirigeants et des contrats d'endettement) de la firme. Il nous semble que même si cette approche s'avère pertinente dans de nombreux cas, elle ne correspond pas à notre volonté d'appréhender le processus de prise de décision relatif au choix d'option concernant la norme IAS 19. Ainsi, le positionnement objectif et les méthodes économétriques généralement utilisées ne répondent pas à l'intention de compréhension des motivations intrinsèques des praticiens lors de la prise de décision comptable. C'est pourquoi, dans le cadre de cet article, nous avons retenu la théorie néoinstitutionnelle sociologique (TNIs) comme grille d'analyse de l'objet de recherche.

L'approche néo-institutionnelle suppose que la survie des organisations est dépendante de son degré de conformité à l'environnement institutionnel (Tatiana Kostova et al., 2008). Si les travaux initiaux de ce courant sont consacrés à l'explication de l'homogénéité des pratiques, les développements ultérieurs mettent en évidence sa capacité à interpréter les situations de changement.

La TNIs permet de mettre en exergue les pressions du contexte socio-économique subies par les groupes dans leur quête de légitimité. L’homogénéité des pratiques peut s'expliquer par l'apparition de comportements isomorphique. L'isomorphisme est défini par Amos Hawley (1968) comme « un processus de contrainte qui force une unité appartenant à une population à ressembler aux autres unités qui sont confrontées aux mêmes conditions environnementales ». Trois types de comportement isomorphique peuvent être identifiés :

- L’isomorphisme coercitif est le résultat de pressions exercées par les différentes institutions sur les organisations. Les instances réglementaires établissent des règles auxquelles les firmes doivent s'adapter ;

- L'isomorphisme normatif fait référence aux pressions qui peuvent exister dans un secteur d'activité. Les organisations sont influencées par des normes professionnelles incitant les membres d'un secteur, d'une branche ou d'une communauté à s'y conformer ;

- L'isomorphisme mimétique renvoie, quant à lui, au comportement en situation d'incertitude. Dans ces conditions, les organisations se livrent au mimétisme en imitant

\footnotetext{
${ }^{6}$ Franck Missonier-Piera (2004), Rick Cuijpers et Willem Buijinik (2005), Joachim Gassen et Thorsten Sellhorn (2006), Samira Demaria et Dominique Dufour (2007).
} 
d'autres organisations considérées comme plus performantes. Au sens institutionnaliste du terme, le mimétisme revêt une dimension temporelle. Les firmes observent les autres organisations et adoptent, avec un léger décalage, les pratiques supposées légitimantes (Pamela Haunschild, 1993). C'est pourquoi W.Richard Scott (2001) estime que des groupes de référence apparaissent, ceux-ci sont imités par des firmes suiveuses souhaitant répliquer les pratiques institutionnalisées.

Les trois types d'isomorphisme permettent d'identifier les pressions institutionnelles subies par les préparateurs des comptes lors de la prise de décision comptable. Ils mettent en évidence les facteurs conduisant les praticiens à opérer de manière similaire, répondant ainsi à un faisceau de valeurs partagées.

Ce courant constitue un axe d'explication des choix comptables au sein des organisations dans lesquelles la maximisation des intérêts personnels des acteurs n'exerce pas une influence prépondérante sur les choix de pratiques comptables (Vivian Carpenter et Ehsan Feroz, 2001). De nombreux auteurs ont su se saisir de ce courant pour expliciter des comportements comptables. Stephen Mezias (1990) explique que le cadre néo-institutionnel sociologique est pertinent pour expliquer les pratiques de reporting financier des groupes appartenant à l'indice FORTUNE 200. À partir de douze cas, Philippe Touron (2006) met en exergue les pressions qui poussent une firme à adopter volontairement avant 1995 des normes alternatives. Elena Barbu (2006) décrit le processus d'harmonisation comptable européen à travers un regard néo-institutionnel. Bernard Colasse et Christine Pochet (2008), quant à eux, analysent le processus isomorphique guidant la création du nouveau CNC. Pour notre part, nous retenons la TNIs et plus particulièrement les trois isomorphismes pour expliquer le processus d'évolution des choix d'options comptables en matière de comptabilisation des écarts actuariels. Cette approche classique de la TNIs vise à mettre en évidence les pressions influençant les décisions comptables mais évidemment, nous ne réduisons pas les processus de choix au seul comportement isomorphique. Ainsi, les intérêts économiques sont aussi des facteurs de prise de décision. Nous estimons néanmoins que les mécanismes d'isomorphisme sont l'expression d'un système de valeurs partagées au sein de la discipline comptable participant à l'adoption de pratiques similaires. 


\section{APPLICATION DE LA NORME IAS 19 PAR LES GROUPES FRANÇAIS}

À présent, nous allons préciser les méthodes de collecte de l'information, puis nous restituerons les pratiques retenues par les groupes du SBF 120 en termes de comptabilisation des écarts actuariels sur une période de cinq exercices.

\subsection{LA COLLECTE DES DONNEES}

L'identification des pratiques comptables des groupes français a été effectuée selon la méthode de l'analyse documentaire. Après avoir collecté les rapports annuels (2004-2008), nous avons, pour chaque groupe composant l'échantillon, identifié la méthode retenue pour comptabiliser les pertes et gains actuariels. La période d'observation débute à la date de transition aux normes internationales, soit le $1^{\text {er }}$ janvier 2004, et se poursuit jusqu'au 31 décembre 2008. Cette période de cinq exercices nous permet d'appréhender les choix comptables français dans le temps et de mettre en perspective leurs évolutions.

L'échantillon est composé des groupes appartenant à l'indice SBF 120 au moment du passage aux normes IAS/IFRS. Le tableau 1 précise la constitution de l'échantillon.

\begin{tabular}{|l|c|}
\hline Échantillon de départ au $1^{\text {er janvier 2004 }}$ & 120 \\
\hline Groupes non conformes aux IAS/IFRS ou Groupes étrangers appliquant les IAS/IFRS & 6 \\
\hline Groupes sortis de la cote ou ayant fusionné & 7 \\
\hline Information indisponible & 1 \\
\hline Échantillon final au 31 décembre 2008 & 106 \\
\hline
\end{tabular}

Tableau 1 : L'échantillon observé

Enfin, pour compléter notre dispositif de recherche, nous avons effectué des entretiens semidirectifs auprès de responsables IFRS de groupes cotés français : Alstom, Aréva, Bull, Groupe Flo, Publicis, Rexel. Les entretiens se sont déroulés en septembre 2007. Ces entretiens visent à appréhender le point de vue des préparateurs des comptes sur l'application des normes internationales et particulièrement sur la norme IAS 19.

\subsection{LA PRATIQUE FRANÇAISE EN MATIERE DE COMPTABILISATION DES ECARTS ACTUARIELS}

Avant même d'appliquer la norme IAS 19, les groupes ont utilisé, au moment de la transition, la norme IFRS 1 «Première adoption des normes IFRS ». Celle-ci permet, entre autres, de retenir des exemptions au principe général de retraitement rétrospectif des comptes. 
Concernant les engagements de retraite, IFRS 1 permet à «un premier adoptant de comptabiliser tous les écarts actuariels à la date de transition aux IFRS, même si par la suite il utilise la méthode du corridor pour les écarts actuariels générés ultérieurement...» (IFRS 1, §20). L'observation de l'application de cette exemption est synthétisée dans le tableau 2 :

\begin{tabular}{|l|c|c|}
\hline \multicolumn{1}{|c|}{$01 / 01 / 2004$} & Nombre de groupes & Pourcentage \\
\hline Comptabilisation de tous les écarts actuariels à la date de transition & 78 & 73,6 \\
\hline Choix de ne pas appliquer l'exemption & 27 & 25,5 \\
\hline Information manquante & 1 & 0,93 \\
\hline Total & 106 & 100 \\
\hline
\end{tabular}

Tableau 2 : Application de l'exemption à IAS 19 permise par IFRS 1

À la date de transition, la majorité des groupes a décidé d'imputer le solde des gains et pertes actuariels aux capitaux propres d'ouverture. Le retraitement rétrospectif des engagements de retraite aurait consisté à recalculer les écarts actuariels depuis l'origine des plans, là où de nombreuses entreprises ne les avaient calculés qu'à compter de la date à laquelle elles ont enregistré des provisions. L'option permet de ne plus comptabiliser au compte de résultat les écarts antérieurs à la transition. Près de $74 \%$ des groupes de l'échantillon ont adopté cette exemption. Le cabinet Mazars (2005, p. 12) estime que les groupes précisant ne pas avoir recouru à cette exemption n'en avaient, dans les faits, pas besoin car ils n'avaient pas différé d'écarts actuariels. Une autre explication repose sur l'impact, généralement négatif ${ }^{7}$, de cette option : certains groupes, dont la situation financière était dégradée, ont renoncé à l'option pour ne pas diminuer dangereusement la situation nette. Ainsi, le responsable consolidation du Groupe A a précisé que l'entreprise était dans une situation délicate entre 2004 et 2005 et qu'il n'était pas envisageable d'imputer des pertes actuarielles de près d'un milliard d'euros car cela aurait conduit le groupe à présenter des capitaux propres négatifs : «les pertes actuarielles représentaient un gros montant qui était hors bilan, que tout le monde connaissait. Mais à l'époque, notre groupe n'allait pas bien du tout, la situation nette était proche de zéro, donc on n'a pas utilisé cette option pour ne pas avoir des capitaux propres négatifs »(Responsable consolidation, Groupe A).

\footnotetext{
${ }^{7}$ La majorité des groupes a constaté des pertes actuarielles, ainsi seulement quatre groupes sur les 78 ayant opté pour l'exemption permise par IFRS 1 font apparaître des gains actuariels (Bonduelle, Géophysique, Jcdecaux, Klepierre). Ernst \& Young (2005) estiment que cette option a globalement eu un impact négatif de 8,2 milliards d'euros sur les capitaux propres des sociétés du CAC 40. D'ailleurs, d'après Mazars (2005, p. 12), l'exemption à IAS 19 est l'option qui a eu le plus d'impacts sur les capitaux propres des groupes français.
} 
Postérieurement à la transition, les groupes doivent appliquer la norme IAS 19 et par là-même se positionner entre trois options quant à la comptabilisation des écarts actuariels. Le tableau 3 présente les choix effectués par les groupes du SBF 120 sur quatre exercices.

\begin{tabular}{|l|l|c|c|c|c|}
\hline \multicolumn{2}{|c|}{} & 2005 & 2006 & 2007 & 2008 \\
\hline \multirow{3}{*}{ Compte de résultat } & \multirow{2}{*}{ Méthode du corridor } & 76 & 64 & 54 & 49 \\
\cline { 2 - 6 } & \multirow{2}{*}{ Intégralement au compte de résultat } & $71,70 \%$ & $60,38 \%$ & $50,94 \%$ & $46,23 \%$ \\
\cline { 3 - 6 } & & 10 & 8 & 9 & 9 \\
\hline \multirow{2}{*}{ Bilan } & \multirow{2}{*}{ Imputation en capitaux propres (SoRIE) } & $9,43 \%$ & $7,55 \%$ & $8,49 \%$ & $8,49 \%$ \\
\hline \multirow{2}{*}{ Information manquante } & $15,09 \%$ & $29,25 \%$ & $38,68 \%$ & $44,34 \%$ \\
\hline
\end{tabular}

Tableau 3 : Comptabilisation des écarts actuariels sur quatre exercices

Tout d'abord, on constate la part relativement faible, mais stable, des groupes ayant opté pour une comptabilisation intégrale des écarts actuariels en résultat. Le faible recours à cette option par les groupes français est conforme aux études européennes menées par Ana Isabel Morais (2008) pour l'exercice 2005 et par le cabinet Ineum Consulting (2008) pour l'exercice 2006.

En 2005, les groupes de l'échantillon se sont majoritairement orientés vers l'option du corridor permettant de comptabiliser seulement les pertes ou gains actuariels au-dessus du corridor. Seulement 16 groupes ont choisi d'imputer directement l'intégralité des écarts actuariels en capitaux propres. Le choix d'une comptabilisation directe dans les fonds propres peut engendrer une certaine volatilité du bilan. Ainsi, puisque les écarts actuariels sont indexés sur la juste valeur des actifs du régime de retraite, la variation de valeur aurait des effets immédiats sur la situation nette des groupes. Par ailleurs, Ernst \& Young (2006) et Ana Isabel Morais (2008) soulignent que le choix du corridor relève d'une contingence nationale. Ainsi, ces études menées en 2005, sur des échantillons européens, mettent en évidence l'adoption généralisée du corridor en France ${ }^{8}$ et en Italie, alors que la Grande-Bretagne et la Hollande ont majoritairement opté pour une comptabilisation directe en capitaux propres.

Dès que l'on se penche sur les exercices ultérieurs, on constate une tendance plus prononcée pour l'adoption de la méthode d'imputation directe des écarts actuariels en capitaux propres. Ainsi, la part de la méthode SoRIE est passée de 15,09\% en 2005 à 44,34\% en 2008, ce qui

\footnotetext{
${ }^{8}$ On peut penser que les groupes ont favorisé la méthode du corridor car elle constitue la méthode de référence prescrite par la recommandation CNC 2003 R-01 (section 6 §6.2.6) en vigueur pour les comptes sociaux, ceci afin de maintenir une unité entre comptes individuels et comptes consolidés.
} 
montre que, petit à petit, les groupes convergent vers l'alternative. Certains groupes justifient ce choix par un souci de transparence de l'information financière : "la direction considère que cette méthode est préférable, dans la mesure où elle donnera une image plus représentative de la juste valeur des actifs et passifs relatifs aux engagements de retraite » (Alcatel, 2007, p.184). Sur la période d'observation, on peut penser qu'un effet de contagion s'est produit. D'ailleurs, selon Romain Appercel (2008), près de la moitié du SBF 120 aurait opté pour l'amendement SoRIE au cours de l'exercice 2008, ce qui correspond approximativement à nos résultats.

Les groupes retenant la méthode SoRIE sont dans l'obligation d'appliquer la norme IAS 8 «Méthodes comptables, changements d'estimations comptables et erreurs » et d'accomplir les travaux relatifs à un changement de méthode. Ainsi, «afin d'assurer la comparabilité des données et conformément à IAS 8, les états financiers des exercices 2006 et 2005 ont été retraités »(Essilor, 2007, p.99).

Le choix d'une option n'est pas anodin sur les états financiers. Ainsi opter pour une comptabilisation directe affecte immédiatement le montant de la situation nette alors que la méthode du corridor a pour effet de lisser et différer la comptabilisation des écarts actuariels. Notons que l'impact sur les capitaux propres de l'amendement d'IAS 19 est modéré pour les groupes ayant retenu l'exemption permise par IFRS 1 permettant la comptabilisation des écarts actuariels antérieurs en capitaux propres à la date de transition. Le tableau suivant met en évidence l'impact de l'adoption de la méthode SoRIE sur les capitaux propres.

\begin{tabular}{|l|c|c|c|c|}
\hline En M€ & $\mathbf{2 0 0 5}$ & $\mathbf{2 0 0 6}$ & $\mathbf{2 0 0 7}$ & $\mathbf{2 0 0 8}$ \\
\hline Moyenne & $-60,41$ & 38,65 & 39,24 & $-121,83$ \\
\hline Écart-type & 38,13 & 66,81 & 65,40 & 217,87 \\
\hline Minimum & -415 & -424 & -207 & -1965 \\
\hline Maximum & 0 & 632 & 612 & 647 \\
\hline
\end{tabular}

Tableau 4 : Impacts de la méthode SoRIE sur la situation nette

Le tableau 4 nous amène à constater une certaine variabilité de l'impact des écarts actuariels comptabilisé en capitaux propres. Si en 2005 l'impact moyen était négatif, il s'est avéré positif pour les exercices 2006 et 2007 ; et avec le retournement des marchés financiers en 2008 on constate un effet négatif sur les capitaux propres au 31/12/2008. Il n'y a donc pas un impact systématiquement négatif ou positif expliquant le choix de la méthode, et ce n'est donc pas au regard d'une explication purement économique que l'on pourra comprendre ce qui pousse les firmes à opter pour cette méthode. 
L'observation de l'application d'IAS 19 sur cinq exercices comptables met en lumière un lent processus d'adoption de l'amendement SoRIE. Néanmoins, on constate un tassement relatif dans la tendance à opter pour la comptabilisation directe en capitaux propres.

\section{UNE INTERPRETATION NEO-INSTITUTIONNELLE SOCIOLOGIQUE DES PRATIQUES COMPTABLES}

L'observation des pratiques comptables des groupes de notre échantillon identifie un recours croissant à l'amendement SoRIE. Nous allons montrer que cette évolution des méthodes comptables peut être appréhendée au regard des apports de la théorie néo-institutionnelle dans son acception sociologique. Pour cela, nous allons mettre en exergue les phénomènes d'isomorphismes coercitif, normatif et mimétique existant au sein du processus de choix d'options comptables.

\subsection{L'IASB EN PRESCRIPTEUR DE CHOIX : LA MANIFESTATION DE L'ISOMORPHISME COERCITIF}

Les organismes de normalisation comptable peuvent être assimilés à des institutions dans la mesure où ils construisent, homogénéisent et reproduisent des attentes standards et, ce faisant, stabilisent l'ordre international (Paul Dimaggio et Walter Powell, 1997). L'IASB est l'institution qui conçoit et diffuse les normes comptables internationales applicables par les groupes européens. Cependant, si cette institution détient un pouvoir réglementaire incontestable, elle n'a pas de pouvoir coercitif direct sur les firmes. Néanmoins, son rôle est primordial pour la comptabilité des entreprises. Même si l'IASB n'a pas d'emprise sur les groupes, contrairement à l'AMF ou au CNC, il exerce un pouvoir coercitif indirect. Ainsi, les projets de normes lancés par le normalisateur aboutiront à plus ou moins court terme à une application sur le territoire français. C'est pourquoi les groupes sont très attentifs aux travaux et publications de l'IASB. Concernant la problématique de la comptabilisation des avantages du personnel, l'influence de l'IASB sur les choix est particulièrement perceptible. La norme IAS 19 régissant les avantages du personnel a été publiée en 2002, elle a fait l'objet de plusieurs amendements (en 2005 et 2007) et depuis le début de l'année 2008, l'IASB a entamé une réforme d'IAS 19. Les groupes sont dès lors extrêmement soucieux de suivre et d'anticiper les évolutions normatives. Pour illustration, nous pouvons citer un extrait du 
rapport annuel du groupe Métropole TV qui justifie de la façon suivante le choix de la méthode SoRIE : «le Groupe, suivant en cela la préconisation de l'IASB, estime que l'option offerte par IAS 19 permet en effet de choisir une méthode plus simple et transparente que celle auparavant utilisée pour présenter les passifs des engagements de retraite » (Métropole TV, 2007, p.166).

D'ailleurs, le projet de révision d'IAS 19 envisage la suppression de l'option du corridor car selon Philippe Danjou (Membre de $\left.1^{1} \mathrm{IASB}\right)^{9}$, « il faut faire en sorte que, à chaque arrêté de comptes, le montant réel du passif soit reconnu au bilan ». Dans ces conditions, le choix de la méthode SoRIE constitue une anticipation normative. À titre d'illustration, le responsable consolidation du Groupe F justifie le choix de l'amendement à IAS 19 par le fait qu'il estime que c'est la méthode préférentielle de l'IASB : «on a hésité à prendre cet amendement. Néanmoins comme ça a été présenté comme la norme du futur, c'est-à-dire celle qui va rester (...) c'est la méthode que nous considérons, par notre interprétation de bureau, comme étant la méthode préférentielle ».

Enfin, l'observation des pratiques montre un tassement relatif dans la tendance à opter pour la comptabilisation directe en capitaux propres (6 groupes ont changé de méthodes entre 2007 et 2008). Ce phénomène doit certainement être considéré comme un comportement attentiste au regard des travaux profonds de réforme de la norme IAS 19 entamés à partir de 2008 par l'IASB. Le choix de la méthode de comptabilisation des écarts actuariels semble donc influencé par les évolutions normatives initiées par l'institution comptable internationale.

\subsection{LE POIDS DE LA PROFESSION SUR LES CHOIX COMPTABLES : L'EFFET DE L'ISOMORPHISME NORMATIF}

Les choix comptables sont intimement liés à l'environnement professionnel des préparateurs des comptes. Les membres de la profession ont un rôle prescripteur fort, conduisant à l'apparition d'un isomorphisme normatif. Ainsi, les consultants, les Commissaires Aux Comptes (CAC) et les associations professionnelles sont un maillon essentiel du processus de choix d'options comptables.

Le responsable normes et procédures du Groupe D considère les CAC comme une aide précieuse, ainsi, «lorsqu'il y a un choix d'option à faire, nous consultons les commissaires aux comptes, qui peuvent nous dire comment ils sentent un peu les choses (...) et ce que font les autres ». L'influence normative des CAC est indiscutable, et les informants en ont

\footnotetext{
${ }^{9}$ Propos recueillis par Alexandra Pétrovic (2008) pour La Tribune.
} 
largement fait part. Dans le cas de la comptabilisation des écarts actuariels, les commissaires aux comptes ont sensiblement «orienté » leurs clients. Le responsable consolidation du Groupe $\mathrm{F}$ précise avoir consulté ses auditeurs avant de retenir définitivement l'amendement SoRIE : «on en a discuté avec nos auditeurs par ailleurs, ils ont dit oui il y a beaucoup de sociétés qui vont le faire ». Selon le même principe, le directeur comptable du Groupe I précise avoir retenu l'option sur le conseil de ses actuaires. Pour le responsable normes et procédures du Groupe G, les CAC constituent une pression dont il faut tenir compte, ainsi, « on a aussi la pression des commissaires aux comptes, qui ont une solution préférentielle qu'ils ont déjà auditée ou fait appliquer par d'autres de leurs clients. Ils nous diront un tel fait comme ça, donc il faut faire pareil, pourquoi voulez-vous faire autrement ? On a aussi ce type de contraintes lors du choix ». Le rôle des professionnels extérieurs à la firme est souligné par Marie-Pierre Peillon (in Nicolas Véron, 2007, p.45) qui affirme que «la pression à l'homogénéisation des pratiques s'effectue avant tout à travers les auditeurs ; quoi qu'on en dise, ces derniers n'aiment guère le cas par cas ». Plusieurs études tendent à mettre en exergue le poids des commissaires aux comptes sur le processus de décisions comptables (Martin Hoogendoorn, 2006 ; David Alexander et al., 2008). D'ailleurs, selon Philippe Touron (2006), les CAC représentent le mécanisme prescripteur de l'isomorphisme normatif.

L'isomorphisme normatif est également véhiculé par les réunions d'associations professionnelles $\left(\mathrm{ACTEO}^{10}\right.$, IMA France ${ }^{11}$, l'Académie ${ }^{12}$, etc.). Celles-ci jouent un rôle important, que ce soit de manière involontaire ou dans le but explicite d'imposer à leurs membres un certain standard (Jabril Bensedrine et Benoît Demil, 1998, p.92). Lors de la transition vers les normes comptables internationales, les associations professionnelles ont eu un réel rôle d'intégrateur de la nouveauté et de partage des connaissances comptables. D'ailleurs, les groupes se sont largement investis dans ces organisations. Dans l'enquête menée par Samira Demaria (2008, p. 18), 56\% des répondants affirment que leur groupe est membre d'une association professionnelle. De plus, $85,8 \%$ des répondants membres d'une

\footnotetext{
${ }^{10}$ ACTEO est une association pour la participation des entreprises françaises à l'harmonisation comptable internationale. Cette association créée en 1997, sur l'initiative du MEDEF, regroupe la majorité des groupes cotés français.

${ }^{11}$ IMA France organise régulièrement des petits-déjeuners thématiques. Récemment, plusieurs ont été consacrés à la norme IAS 19: L'actualité IAS 19 et IFRS 2: sur les avantages au personnel, le 27 mai 2008, Gestion prospective des engagements de retraites le 1 avril 2008 ; IAS 19: présentation du discussion paper, le 3 mars 2009.

${ }^{12}$ L'Académie des Sciences et Techniques Comptables et Financières a organisé, le 22 septembre 2008, une conférence sur le thème «Retraites et fonds de pension : Les évolutions à court terme d'IAS 19 proposées par l'IASB ».
} 
association se rendent régulièrement aux manifestations organisées par la profession : entre une fois par mois et une fois par trimestre.

Les groupes de réflexions constitués par les associations ont un véritable pouvoir prescripteur sur les praticiens. Ainsi, les responsables consolidation des Groupes A et F expliquent s'être informés lors des différentes commissions d'ACTEO et des réunions des cabinets d'audit afin d'identifier les pratiques reconnues par la profession : «on a hésité à prendre cet amendement là... alors on a écouté ce que faisaient les autres dans des commissions ACTEO et compagnie » (Responsable consolidation, Groupe F). De même, le responsable consolidation du Groupe A précise : «j'ai envoyé quelqu'un de mon équipe, c'était en juillet [2007], justement sur IFRS 5 et l'amendement à IAS 19 : retour d'expérience».

En somme, il apparaît que le processus de décision comptable est fortement influencé par les pressions exercées par les professionnels externes aux groupes. Les préparateurs des comptes se positionnent en fonction de leurs pairs intégrant dès lors des pratiques reconnues par la profession. Dans ces conditions, retenir l'amendement SoRIE s'apparente à un choix teinté d'isomorphisme normatif conduisant à une homogénéité des pratiques au regard du champ disciplinaire.

\subsection{LA GENERALISATION DU BENCHMARK ET DE LA VEILLE : L'EXPRESSION DE L'ISOMORPHISME MIMETIQUE}

Le dernier axe de compréhension du processus de choix comptable repose sur l'isomorphisme mimétique qui s'articule autour des stratégies de benchmark et de veille comptable. Le benchmark correspond à une approche structurée visant à identifier les meilleures pratiques d'une industrie ou d'un secteur, afin de comparer et d'adapter les processus d'une organisation. Jabril Bensedrine et Benoît Demil (1998, p. 104) estiment que «le benchmarking apparaît clairement comme l'institutionnalisation d'un processus mimétique, puisqu'il consiste de façon générale à se comparer aux concurrents et à s'inspirer éventuellement de leurs recettes ». Avec l'adoption des normes IAS/IFRS, les équipes comptables ont massivement mis en œuvre le benchmark et la veille comptable. Ces pratiques constituent une manifestation de l'isomorphisme mimétique. Les sociétés s'observent, comparent leurs pratiques et adoptent la méthode qu'elles estiment reconnue par la communauté. Ce processus n'est pas instantané, il relève d'une volonté prolongée de se comparer et de se conformer aux firmes appartenant au même champ organisationnel. Les deux stratégies introduisent un délai entre l'observation et la mise en application des 
méthodes observées, intégrant ainsi une dimension temporelle propre à l'isomorphisme mimétique, conformément aux critères de Pamela Haunschild $(1993)^{13}$. Selon le directeur comptable du Groupe H, un système comptable n'est efficace et performant que s'il anticipe la réglementation, et pour cela, la veille comptable est essentielle. Ainsi, d'après lui, «ce qui fait le sérieux d'un groupe, c'est l'existence d'une veille technologique, c'est-à-dire un département, un responsable des procédures IFRS et US GAAP, c'est-à-dire quelqu'un qui ne s'occupe que de ça». Le responsable normes et procédures du Groupe C estime que la veille comptable est particulièrement utile lorsque le groupe est confronté à une option : «quand on se trouve face à un problème que l'on découvre, on aime bien savoir comment les autres professionnels réagissent, donc c'est vrai qu'on s'est renseigné pour savoir si d'autres avaient le problème, s'ils avaient déjà réfléchi dessus et comment ils le traitaient ».

Le choix de la méthode de comptabilisation a donc largement reposé sur un processus d'imitation, les préparateurs des comptes s'observant mutuellement et se décidant lorsque des firmes de référence ont officialisé une position. Le responsable de la consolidation du Groupe A décrit exactement ce processus : «concernant la méthode SoRIE, toutes les sociétés sont en train de faire comme nous, c'est à dire de changer de méthode sur ce sujet là. Il y a eu Suez et Accor qui l'ont fait dès 2006, Publicis l'a fait en juin. Parce qu'aujourd'hui, tout le monde se dit: le plus simple c'est de tout passer en situation nette, comme ça il n'y a plus d'impact au compte de résultat pour les pertes et gains actuariels ». De même, le responsable consolidation du Groupe F précise que, lors des discussions avec son directeur financier sur l'opportunité de retenir l'amendement SoRIE, le fait que des sociétés importantes du CAC aient déjà opté pour ce choix a pesé sur la décision. Ce comportement imitatif illustre la dialectique entre firmes de référence et firmes suiveuses. Ainsi, une quinzaine de groupes ont adopté dès 2005 une comptabilisation intégrale des écarts actuariels en capitaux propres (Havas, Ingenio, PPR, Valéo...) et les autres groupes ont pris le temps d'observer et d'analyser les effets de l'option, puis, décide de la suivre ou pas. On constate que des vagues successives d'adoption ont eu lieu entre 2005 et 2008, puisqu'environ une dizaine de groupes optent annuellement pour la méthode SoRIE.

La veille comptable et l'activité de benchmark conduisent les groupes à adopter des options similaires. Ce comportement est cohérent avec les propos de Philippe Touron (2006) selon lesquels les groupes en quête de légitimité ajustent leurs décisions selon une rationalité

\footnotetext{
${ }^{13}$ Les trois critères énoncés par Pamela Haunschild (1993, p. 567) pour identifier l'isomorphisme mimétique sont 1) Il doit exister une méthode de référence appliquée par des entreprises du champ organisationnel ; 2) Les praticiens doivent être exposés au modèle ; 3) La firme adopte la méthode en retard.
} 
collective, instaurant une conformité isomorphique. Les pressions institutionnelles induisent un isomorphisme mimétique poussant les firmes à s'imiter les unes les autres afin de gagner en légitimité (Jacob Broadbent et Richard Laughlin, 2001).

\section{CONCLUSION}

Cet article met en lumière le recours croissant à l'amendement SoRIE par les groupes français. L'étude d'un échantillon de 106 firmes appartenant au SBF 120 sur une période de cinq exercices souligne l'évolution constante de cette méthode par rapport aux alternatives envisageables. Nous interprétons cette tendance au regard des apports de la théorie néoinstitutionnelle sociologique, en identifiant les trois isomorphismes lors du processus de choix de la méthode de comptabilisation des écarts actuariels sur les prestations de retraite.

Le recours à la comptabilisation en capitaux propres des pertes et gains actuariels contribue à introduire une dose supplémentaire de juste valeur au bilan et, par là-même, un niveau de volatilité potentiellement défavorable pour le groupe. Ainsi, Robert Obert (2008) souligne que la crise financière a des effets sur de nombreuses normes utilisant la juste valeur de manière partielle comme IAS 19. D'ailleurs, dès les prémisses de la crise (septembre 2007), certains informants (responsable consolidation du Groupe A et responsable normes et procédures du Groupe G) soulignaient les effets considérables que pouvaient avoir les fluctuations boursières sur la valeur des capitaux propres. La dégradation du système financier mondial peut avoir des conséquences inattendues sur les états financiers des groupes ayant opté pour ce modèle comptable. Ainsi, Sanofi-Aventis $(2008)^{14}$ précise dans son rapport semestriel les effets négatifs de la crise financière sur les engagements de retraite comptabilisés en capitaux propres. Lors de ses recommandations pour l'arrêté des comptes, l'AMF (2008) souligne l'influence de la détérioration des marchés financiers sur le montant des engagements de retraite, en particulier si le groupe enregistre immédiatement les écarts actuariels au résultat ou en capitaux propres. Il sera intéressant de mesurer les effets de la crise financière sur les sommes comptabilisées par les groupes en termes d'écarts actuariels, et cela au regard de l'option retenue. Enfin les travaux en cours au sein de l'IASB semblent privilégier une approche complètement nouvelle de la comptabilisation des écarts actuariels, en opérant une répartition obligatoire entre compte de résultat et bilan. Dès lors même si ces orientations ne

\footnotetext{
${ }^{14}$ Plusieurs groupes de l'échantillon ont fait des commentaires similaires, c'est le cas entre autres de Valeo et de Véolia.
} 
constituent que les prémisses d'une future norme, on peut se demander si les groupes ne vont pas s'en inspirer pour l'établissement des états financiers des années à venir.

\section{BIBLIOGRAPHIE}

David Alexander, Brigitte Eirle et Christoph Hutten. The auditor as standards-setter : some US evidence and its implications. $29^{\text {ème }}$ Congrès de l'AFC, 2008. ESSEC Cergy-Pontoise.

AMF. Études de l'information fournie par les sociétés du CAC40 sur les engagements de retraite et assimilés. 2004.

AMF. Recommandations de l'AMF en vue de l'arrêté des comptes 2008. 2008.

Romain Appercel. Les engagements sociaux et IAS 19. Actuaria CNAM, 2008.

Elena Barbu. Les entreprises françaises cotées face à l'harmonisation comptable internationale : une analyse néo-institutionnelle d'un long processus vers l'homogénéité. Thèse en sciences de gestion, 2006, Université d'Orléans.

Jabril Bensedrine et Benoît Demil. L'approche néo-institutionnelle des organisations in Repenser la stratégie : fondements et perspectives, Laroche et Nioche, 1998. Paris. p. 85-110. Jacob Broadbent et Richard Laughlin. Organizational resistance strategies to unwanted accounting and finance changes. Accounting, auditing and accountability journal, 2001, vol. 14. $n^{\circ}$ 5. p. 565-586.

Alexis Canuet. Normes comptables contre fonds de pension. Alternatives économiques, 2007, vol. n²56. p. 72-73.

Vivian Carpenter et Ehsan Feroz. Institutional theory and accounting rule choice : an analysis of four US state governments' decisions to adopt generally accepted accounting principles Accounting, Organizations, and Society, 2001, vol. 26. nº7-8. p. 565-596.

CNC. Preliminary Views on Amendments to IAS 19 Employee Benefits. 2008.

Bernard Colasse et Christine Pochet. De la genèse du nouveau conseil National de la Comptabilité (2007) : un cas d'isomorphisme institutionnel ? $29^{\text {ème }}$ congrès de l'AFC, 2008. ESSEC Cergy-Pontoise.

Rick Cuijpers et Willem Buijinik. Voluntary adoption of non local GAAP in the European Union : a study of determinants and consequences. European accounting review, 2005, vol. 14. n³. p. 487-524.

Cécile Darche et Emmanuelle Levard-Guilbault. IAS 19 : Evolutions à court terme. conférence IMA France, 2009.

Samira Demaria. Changement de normes : la stabilité des choix comptables. $29^{\text {ème }}$ Congrès de l'AFC, 2008. ESSEC Cergy-Pontoise.

Samira Demaria et Dominique Dufour. Les choix d'options comptables lors de la transition aux normes IAS/IFRS : quel rôle pour la prudence ? Comptabilité contrôle audit, 2007, vol. Thématique. ${ }^{\circ}$ Décembre. p. 195-218.

Paul Dimaggio et Walter Powell. Le néo-institutionnalisme dans l'analyse des organisations (Traduction de l'introduction de l'ouvrage de 1991). Politix, 1997, vol. 10. n40. p. 113-154.

Ernst \& Young. Passage aux ifrs : les pratiques des grands groupes européens. Ed. CPC Meylan: 2005. 
Ernst \& Young. Observations on the implementation of IFRS. 2006.

Joachim Gassen et Thorsten Sellhorn. Applying IFRS in Germany : determinants and consequences. Available at SSRN: http://ssrn.com/abstract=906802, 2006.

Pamela Haunschild. Interorganizational imitation: the impact of interlocks on corporate acquisition activity. Administrative science quarterly, 1993, vol. 38. n4. p. 564-592.

Amos Hawley. Human ecology in International encyclopedia of the social sciences, Ed. Mcmillan, 1968. New-York. p. 328-337.

Martin Hoogendoorn. International accounting regulation and IFRS, implementation in Europe and beyond-experiences with first time adoption in Europe. Accounting in Europe, 2006, vol. 3. $\mathrm{n}^{\circ} 1$. p. 23-26.

IASB. Discussion paper : Reducing complexity in reporting financial instruments. 2008.

Ineum Consulting. Evaluation of the Application of IFRS in the 2006 Financial Statements of EU Companies. Report to the European Commission, 2008.

Tatiana Kostova, Kendall Roth et Tina Dacin. Institutional theory in the study of multinational corporations : a critique and new directions. Academy of management review, 2008, vol. 33. n ${ }^{\circ}$. p. 994-1006.

Mazars. IFRS : la communication financière des groupes français en 2004. 2005. Les cahiers Mazars.

Stephen Mezias. An institutional model of organizational practice : financial reporting at the fortune 200. Administrative science quarterly, 1990, vol. 15. n¹990. p. 431-457.

Franck Missonier-Piera. Economic determinants of multiple accounting method choices in a Swiss context. Journal of international financial management and accounting, 2004, vol. 15. n². p. 118-144.

Ana Isabel Morais. Actuarial gains and losses: the choice of the accounting method Accounting in Europe, 2008, vol. 5. ${ }^{\circ}{ }^{2}$. p. 127-139.

Robert Obert. Les IFRS sont-elles la cause de la crise financière? Revue française de comptabilité, 2008, vol. 415. n Novembre. p. 43-45.

Alexandra Pétrovic. La comptabilité des passifs sociaux bientôt revue. La Tribune, 2008.

RF Comptable. Les engagements de retraite et avantages similaires. 2003.

W.Richard Scott. Institutions and organizations. Ed. SAGE: 2001.

Danielle Sougné. L'évaluation actuarielle des engagements de pension selon l'IAS 19 et ses perspectives d'évolution. Revue française de comptabilité, 2009, vol. 240. n Avril. p. 44-50.

Philippe Touron. Douze cas d'adoption de normes comptables alternatives sur la période 1970-1995 : de la théorie de l'agence à la légitimité institutionnelle. Thèse en Sciences de Gestion, 2006, HEC.

Nicolas Véron. Points de vue d'investisseurs sur la mise en oeuvre des normes IFRS. 2007. AFG \& FFSA. 